\title{
MICMOC/MICMOS: Photochemistry of van der Waals solids and the rise of the organic molecular complexity
}

\author{
L. Le Sergeant d'Hendecourt ${ }^{1}$, P. de Marcellus ${ }^{1}$, P. Modica ${ }^{1}$ \\ Astrochimie et Origines, IAS-CNRS-UPS, UMR 8627, Campus d'Orsay, bat 121, 91944 Orsay, \\ Cedex, France
}

\begin{abstract}
Ices of well-known composition are widely observed in molecular clouds out of which stars, planets and debris (asteroids, comets) will form. These ices are naturally subjected to energy input in the form of UV photons and charged cosmic particles which are able to start a very rich radical chemistry in the solid state. These phenomena are simulated in the MICMOC experiment which focuses on the possible prebiotic significance of the organic residues that are formed at room temperature. Further than MICMOC, we propose a general prospective of the evolution of this experiment toward the concept of non-directed experiments that may allow simulating the very first steps from the inanimate molecular world to selective pathways toward self-replicating autocatalytic and heterotrophic molecules that could be considered, for a physicist a template for "minimal" life.
\end{abstract}

\section{Interstellar ices}

Interstellar ices are well known since a very long time, thanks to the advent of infrared spectroscopy in astronomy in the mid 70's. However, it was not until 1979 that water ice was shown to indeed explain the strong and very broad absorption band observed in the BN-KL object in Orion [1]. Note that a precise identification of this band relies on dedicated laboratory experiments where it could be shown that the IR spectrum of the $\mathrm{OH}$ band from amorphous $\mathrm{H}_{2} \mathrm{O}$ ice did match closely the observed astronomical band [2]. The development of simultaneous astronomical observations in the IR region, in particular from airborne telescopes such as the KAO [33] and larger ground based ones together with the extensive use of laboratory simulations led quickly to the discovery of the solid CO molecule [4], whose observation could also be interpreted in terms of grain temperatures [5], based on the thermodynamic properties of these ices. Finally, the growth of infrared astronomy with IRAS, ISO and Spitzer satellites did allow the detection of many and abundant species in the solid phase, under the generic name "interstellar ices", mostly in protostellar objects where ices are detected in the collapsing molecular cloud material that is located in front of the IR source formed by the interaction of the protostar with the infalling material. A list of interstellar ice molecules with their abundances, 
respective to the water molecule (100) is given in Figure 3. Note that, because of grain accretion of the gas phase species, solid state molecules $\left(\mathrm{H}_{2} \mathrm{O}, \mathrm{CO}, \mathrm{H}_{2} \mathrm{CO}, \mathrm{CH}_{3} \mathrm{OH}\right)$, in a given line of sight, always dominate in abundances their gas phase counterparts by one to three orders of magnitude. An excellent review on icy molecules can be found in [6] from which Figure 1 is extracted, and complementary information can be obtained in [7].

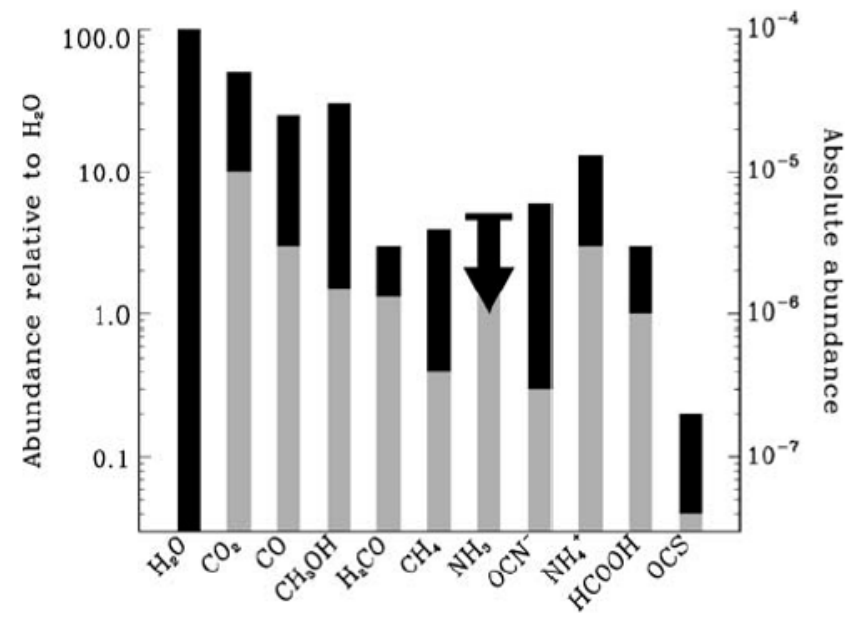

Figure 1: Abundances of observed interstellar ices relative to $H 2 O$. The black extension does indicate variations of these relative abundances from source to source (from [34]).

Before to conclude this short introduction on ices, let me recall that only dedicated and careful laboratory experiments able to replicate the observed spectra have allowed demonstrating the intricate nature of these ices. For example, the formation of molecular complexes (e.g. $\left.\mathrm{CO}_{2}: \mathrm{CH}_{3} \mathrm{OH}\right)$ or hydrates such as ammonia hydrates $[8,9]$ has been established and explained in the laboratory and the corresponding features (band shapes in particular) in astronomical spectra have been duly observed. Thus, and most importantly for the irradiation experiments described in the following sections, the nature of the starting mixtures of the used laboratory analogs are well enough known that simulation experiments can be used as templates to explain the growing nature of complexity in the organic material on the surface of interstellar grains.

\section{Astronomical evidence for energetic and thermal processing of ices}

Although always debated in the literature, energetic processing of interstellar ices from UV photons and cosmic ray particles has been investigated as early as in the mid 80's. Truly speaking, few direct evidences do exist from the detection of some important intermediate molecules. One of the problems encountered by IR spectroscopy is its absence of sensitivity relative to radio-astronomy, in particular because, in the solid state, many lines are blended or hidden by molecular interactions between a given molecule and the dominant and very polar species, $\mathrm{H}_{2} \mathrm{O}$. Thus it is practically impossible to record molecules that are present under the level of a few percent that of $\mathrm{H}_{2} \mathrm{O}$. Nevertheless, in 1989, the discovery of the solid state $\mathrm{CO}_{2}$ molecule, a direct by-product of the UV photochemistry of ice mixtures involving water and carbon monoxide was obtained using the LRS 
(Low Resolution Spectrometer) instrument onboard IRAS [10]. Remarkably, this discovery was made possible because of model predictions from 1985 [11] examining gas/grain interaction in dense clouds as well as laboratory simulations specially developed to study the effect of UV photochemistry on interstellar ices [12]. The fact that the presence of interstellar $\mathrm{CO}_{2}$, totally absent and unexpected in the models of gas phase chemistry, because its main formation mechanism is endothermic, was indeed predicted from laboratory experiments, has been largely ignored until its "rediscovery" in 1996 by the ISO-SWS instrument onboard ISO [13]. Other solid phase molecules can also be attributed to energetic processing, where UV photons have usually been more employed than cosmic rays, although it should be stressed that the effects of cosmic rays are remarkably similar, as far as the build-up of more complex molecules is considered (see for example [14]).

Actually, as already mentioned, observation of such processes is difficult because of intrinsic properties of water ice making it difficult to observe small abundances species but also because, in a molecular cloud, cold ices in the same line of sight, do mask very efficiently warmer regions where ice sublimates and where, as a result of slow warming up of the grains, for example in hot molecular cores [15], it is nearly impossible to observe the organic material that may remain, as in the laboratory experiments, at the surface on the grains, where all the organic complexity may be found. A counterexample of this situation lies in the detection of the ammonium cyanate bands $\left(\mathrm{NH}_{4}{ }^{+} \mathrm{OCN}^{-}\right.$ ), easily observed in the laboratory and found in the 3 micron region in only one object, MonR2IRS9 where the water ice exhibits a crystalline feature and is clearly evaporating [16].

Other attempts have been performed to detect the effect of UV photolysis with some tentative successes such as in NGC7538-IRS9 where the 5 to 8 micron region may be interpreted by the presence of semi complex molecules such as urea, formamide and glycerol [17]. Although the evidence is scarce, from many bias in the observations, the good knowledge of the molecular composition of interstellar ices and the presence of energetic processing allow experimentalists to use ice evolution, from photo and thermo chemistry, in the laboratory as templates for what may be possible in the interstellar medium, with further evidence for a very complex organic chemistry whose nature is described in the next section.

\section{From ices to organic residues}

Laboratory experiments on interstellar ice analogs are based on a rather simple experimental concept, matrix isolation spectroscopy [18]. A gas mixture is prepared in a metallic high vacuum line where gas phase molecules mimicking those observed towards protostars (e.g. $\mathrm{H}_{2} \mathrm{O}, \mathrm{CH}_{3} \mathrm{OH}, \mathrm{NH}_{3}$, $\left.\mathrm{CO}_{2}, \mathrm{CO} \ldots\right)$ are mixed. This mixture is then deposited onto a glass window cooled to the desired temperature $(10-80 \mathrm{~K})$. A microwave discharge lamp produces an intense UV photons flux, with a given spectrum [19], mostly $\mathrm{H} \alpha$ (but not only). These experiments are largely described in [20, 21, 22] and we shall not come back on the details of those. Usually, to obtain a thorough photolysis of the bulk of the ice, the mixture is deposited together with the photon flux (simultaneous irradiation) so that the building sample does not appear to be thick in the UV. Thus it is possible, in principle, to adjust the ratio of the number of UV photons to the number of deposited molecules. For most experiments this number is adjusted to one but may actually vary from 0.1 to 10 about. One of the interesting points about such a simulation is that this constitutes, in its definition, a non-directed experiment, similar to the one of Miller [23] and thus a "cosmic generalization of Miller's experiment, not limited to planets but to materials which ultimately form them. The knowledge of the ice composition, the environment in which these ices evolve, the subsequent heating when closing a star, for example, in hot core regions where more complex molecules are observed to evaporate from grains, all these processes can be empirically reproduced in the laboratory so that the evolution of the molecular matter can then be followed by sophisticated analytical techniques such as gas chromatography and mass spectrometry (GC-MS) and many other methods that will be used to analyze the organic residues that are formed during these processes. Note that, in this empirical approach, the evolutionary time of the ice can be easily accelerated since a week experiment corresponds roughly to the lifetime of a molecular cloud where the interstellar ices do evolve. At the 
end of the experiment, thus at $300 \mathrm{~K}$, an organic residue is always left on the sample, a semirefractory material that may cover the surface of most interstellar grains, especially in star forming regions. For the sake of simplicity, we present, in Figure 2 a typical in-situ IR spectrum of such a residue coming from the UV photolysis of an ice mixture composed of $\mathrm{H}_{2} \mathrm{O}, \mathrm{CH}_{3} \mathrm{OH}$ and $\mathrm{NH}_{3}$ in a ratio around 2/1/1. Note that these experiments, duplicating the recent ones from [24] are made at a temperature of $80 \mathrm{~K}$ to further speeding up the reaction kinetics (diffusion) in the samples. The ice composition used in the laboratory experiments is close to their compositions in high mass stars molecular regions although $\mathrm{CO}$ and $\mathrm{CO}_{2}$, easily made during photolysis, are not included in this mixture. Many other similar experiments have been performed on these ice photolysis simulations.

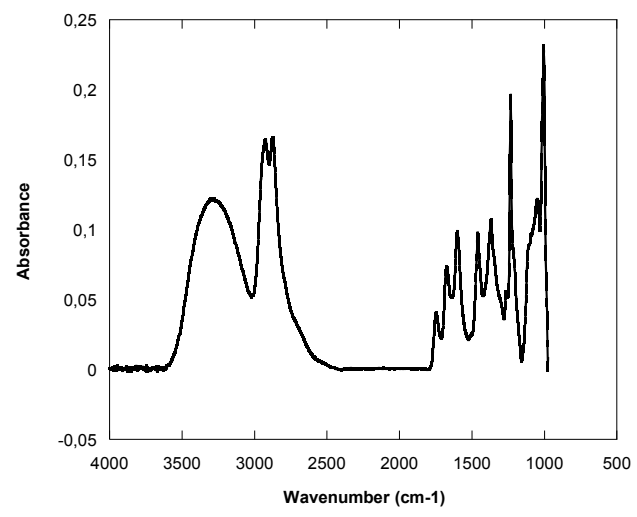

Figure 2: Infrared spectrum of a typical organic residue obtained from interstellar ice analog photochemistry, after evaporation, at room temperature (from [24]).

The characteristic features of these semi-organic residues can be seen from their infrared spectra which are rather insensitive to the precise ice composition (given the fact that the used mixtures are generally similar) as well as to the temperature of deposition (from 10 to $80 \mathrm{~K}$ ). An interpretation of the residue spectrum is given in Table 1 where only chemical functions can be identified, besides the HMT molecule already mentioned in 1995 [25].

Table 1: Infrared bands of the organic residue with their chemical identification.

\begin{tabular}{|c|c|c|}
\hline Position $\left(\mathrm{cm}^{-1}\right)$ & Position $(\mu \mathrm{m})$ & Carrier (mode) \\
\hline $3600-3000$ & $2.78-3.33$ & $\begin{array}{l}\text { alcohols, carboxylic acids (O-H stretching) } \\
\text { and amines (N-H stretching) })^{a, b}\end{array}$ \\
\hline $2957-2945$ & $3.38-3.40$ & carbon chains $\left(\mathrm{CH}_{3}\right.$ stretching) \\
\hline $2926-2923$ & 3.42 & $\begin{array}{l}\text { HMT }\left(2 v_{19}, v_{2}+v_{19}\right)^{c} \\
\text { and aliphatic chains (asym. } \mathrm{CH}_{2} \text { stretching) }{ }^{b}\end{array}$ \\
\hline $2875-2872$ & 3.48 & $\begin{array}{c}\mathrm{HMT}\left(\mathrm{CH}_{2} \text { stretching } v_{18}\right)^{c}, \mathrm{NH}_{4}^{+}\left(2 v_{4}\right)^{a} \\
\text { and aliphatic chains (sym. } \mathrm{CH}_{2} \text { stretching) }{ }^{b}\end{array}$ \\
\hline $2150^{*}$ & 4.65 & nitriles $(\mathrm{C} \equiv \mathrm{N}$ stretching $) ?$ \\
\hline $1750-1745$ & $5.71-5.73$ & esters $(\mathrm{C}=\mathrm{O} \text { stretching })^{a}$ \\
\hline $1675-1665$ & $5.97-6.00$ & amides $(\mathrm{C}=\mathrm{O} \text { stretching })^{a, c}$ \\
\hline $1603-1599$ & $6.24-6.25$ & amides, $\mathrm{NH}_{2}$ including POM ${ }^{a, c}$ \\
\hline $1461-1458$ & $6.84-6.86$ & $\begin{array}{l}\mathrm{NH}_{4}^{+}\left(v_{4}\right)^{a} \text { and } \\
\text { methyl and methylene groups (C-H bending) }{ }^{c}\end{array}$ \\
\hline 1370 & 7.3 & HMT $(\mathrm{C}-\mathrm{H} \text { scissoring })^{c}$ \\
\hline $1235-1233$ & 8.1 & HMT (C-N stretching $\left.v_{21}\right)^{c}$ \\
\hline 1006 & 9.94 & HMT (C-N stretching $\left.v_{22}\right)^{c}$ \\
\hline
\end{tabular}


Actually, many other studies pertain to this organic residue. Among them [27] is one of the most extended and pertinent. The IR spectrum displayed here is very generic and does not change much when similar, but somewhat different relative compositions are considered. These semi-refractory residues are highly functionalized and thus remain almost totally soluble in water, an important characteristic for their availability to a possible further prebiotic evolution if arriving in an ocean on a telluric planet.

\section{Relevance of organic residues to the problem of prebiotic materials}

Before we describe the possible relationship between these organic residues and organic matter found in meteorites, it is important to emphasize that acid hydrolysis treatment of such residues, a classical treatment employed to break up the original macromolecules present in them, has held as many as 16 amino-acids [26, 27] as well as carboxylic acids [28]. Very recently, di-petides have been found in such residues [29]. Although the analytical method is somewhat criticized by its unrealistic parameters $\left(100^{\circ} \mathrm{C}\right.$ in $\left.6 \mathrm{M} \mathrm{HCl}\right)$, it can be argued that this procedure just speeds up the hydrolysis mechanism that will take much more time in a primitive but warm ocean. However, in that case, time is indeed not such an issue since millions of years will be available for this hydrolysis in a primitive ocean and further possible chemistry which may then be really called prebiotic.

In the frame of the cosmic evolution of solid materials depicted as the famous dust cycle in the Galaxy, collapsing molecular cloud materials will, at least partially, end up in planetary disks and may be integrated as asteroids or comets. CM meteorites such as Murchison do contain appreciable amounts of carbon (up to 4\% r.w.t.). Among this fraction, about a fourth is in the form of a soluble organic material whose molecular composition has been previously studied [30]. To which extent is there a link between this soluble organic matter (SOM) in meteorites and the organic residue is not yet precisely known but is the subject to intensive studies. Formerly suspected to be the result of contamination and Earth's weathering, new and more sensitive analytical techniques may help to resolve this issue. As well known, even primitive meteorites have known an episode of thermal and aqueous metamorphism so that a study of its effect on original and genuine organics obtained from photochemical reactions as described above may also be considered and can be easily simulated in non-directed experiments similar to the ones related to extraterrestrial ices evolution.

Recently, it has been reported that irradiation of ice analogs using Circularly Polarized UV light on the DESIRS beamline on the synchrotron radiation facility SOLEIL in France, has produced a proteic amino acid, alanine, with a slight but significant enantiomeric excess, similar to the ones observed in some primitive chondrites from the solar nebula [31]. In the framework of a plausible astrophysical scenario, it is then proposed that a direct link between organic materials from primitive chondrites with photochemistry of interstellar ices exists, including a possible photochemistry step using some CPL whose existence in regions of high mass star formation regions is now documented [32]. One argument from this observation is the fact that CPL light of the same helicity (L or R) is indeed present over large spatial areas in such regions, areas that are much larger, by roughly a factor 100 , than the size of a "solar" nebula. Such an encouraging result relies also on the fact that the experiments using CPL light have used exactly the same parameters than the more classical photochemical ones using non polarized UV light. Thus, not only a prebiotic material may be produced in this way, but it may also contain the chiral asymmetry that is important later from the starting of biochemical reactions [33], see also C. Meinert et al., this volume.

\section{Non directed experiments: a passage from astrochemistry to "astrobiology"?}

Astrochemistry experiments, particularly those related to the study of the evolution of ices, following energetic processes have originally been undertaken to interpret astronomical spectra, especially, 20 years ago, those expected from the ISO satellite whose spectral capabilities opened a new window of observations for the chemistry of molecular clouds [34]. This approach has been altogether very 
successful to understand the global ice composition as well as the importance of their evolution upon irradiation with UV photons and cosmic rays of energies around the $\mathrm{MeV}$. Definitely in the laboratory, a complex organic material is produced, containing hydrosoluble macromolecular fractions. Such experiments may be viewed as a generalization, at the cosmic scale, of Miller's experiment [23] that pertained only to the primitive Earth's atmosphere. However, the possible dominance of an oxidizing atmosphere (rich in $\mathrm{CO}_{2}$ ) is a serious challenge for Miller's hypothesis, even though if his experiment remains chemically pioneering one. This problem may be overcome by the late bombardment of the Earth by a reduced form of carbon that may be found in meteorites or better micrometeorites. Such as Miller's experiment, photochemistry experiments on interstellar ice analogs belong to this class of experiments that may be called non directed ones. Indeed, what is controlled in these experiments is the natural evolution to which some "trivial" and largely observed interstellar matter (ices) is subjected. For example, photon fluxes used in the laboratory allow increasing the kinetics of the reactions so as to obtain in a few days an evolved material that may be very similar to the one that entered the solar nebula after $10^{5 / 6}$ years of evolution. Note that, to the contrary to gas phase networks and reaction rates which are used to study gas phase chemistry in molecular clouds, solid state photochemistry of ices cannot be treated in a totally mechanistic way. Almost 60 years after Miller's work, the full complexity of the byproducts of his rather simple experiment has still not been fully elucidated. The same will be true for the organic residues. Many analytical tools pertaining to physical chemistry will be needed to at most understand the outcome of the experiments and the precise nature of the complex organics formed. A complete reductionist approach of the entire reaction network involved in this operation will be truly impossible.

As far as the "prebioticity" of this material, one must carefully relate this property to the interaction between the organic matter produced in a more than plausible astrophysical scenario with an environment that will be the one of the primitive Earth which is the only example we know where life appearance is undeniable. Fortunately, the progressive knowledge of the primitive Earth [35] is entering a new phase where hypotheses about the nature of the early atmosphere and ocean on the Earth may be made in a more precise context. This will allow to study the interaction of some organics with a peculiar environment so that a phase space of parameters $(\mathrm{T}, \mathrm{pH}$, illumination...) may be explored using the semi-empirical approach of a non-directed experiment. For example, the mechanism for formation of oligo-peptides [36, 37] that may be considered as, yet small, protoproteins, provides a very useful framework to be tested in the laboratory. If such oligo-peptides can be synthesized by very natural processes in the laboratory, the route to PNA's (Poly-Nucleic-Acids) may be possible [38]. Such molecules are believed to be capable of some autocatalytic properties, thus defining a very "minimal" form of life (autocatalytic and heterotrophic capabilities), that may be (or not) connected to some form of Darwinian type evolution which may later imply the building of a simple genetic code However, such non directed experiments may help astrochemistry to really enter the field of "astrobiology" by offering at least a semi-empirical but practical explanation between the molecular universe and the very early (pre)biochemical one. Besides, very recent work popularized in [39] may open a new field in this complex subject where life is not the result of a unique process but may be viewed as a succession of irreversible events out of thermodynamic equilibrium involving more and more complex autocatalytic replicators whose stability is based on Dynamic Kinetic stability of these replicators. Prebiotic chemistry is actually in its infancy.

\section{Conclusions}

Based on astronomical arguments i.e. the availability of the elements, due to their cosmic abundances and their physic-chemical properties, we point the fact that the basic elements for life supporting systems, $\mathrm{H}, \mathrm{O}, \mathrm{C}, \mathrm{N}, \mathrm{S}$ and $\mathrm{P}$, the ones that are present in DNA, cannot seriously be different elsewhere. Moreover, the very special chemical properties of carbon offer an extremely versatile and rich organic chemistry that is of considerable interest for life if associated with liquid water for its role as a solvent and transport. For a chemist, the number of organic molecules is virtually "infinite" a fact that is certainly determinant for prebiotic chemistry and early biochemical 
reactions. Recall that the number of minerals known to date is just around... 4500. Thus organic chemistry will provide a real continuum of species which must be at the heart of the Darwinian evolution. Indeed, if mutation, adaptation and selection are the most important characteristics of living systems, such a very progressive and small step by step evolution requires this chemical continuum to ensure that these three characteristics are always met.

Thus life based on very different mechanisms is highly improbable and the focus on the studies, connecting astrochemistry, complex organics that arise on extraterrestrial solids and end up in primitive oceans where prebiotic chemistry will start and may lead to the rise of the first biomolecular reactions, must be seriously considered. This field, accessible to experimentation, must be viewed as an interdisciplinary field strongly unified by cosmic evolution.

\section{References}

1. Soifer, B.T., Puetter, R.C., Russell, R.W., et al., Astrophys.J. 232, L53-L57 (1979)

2. Léger, A., Klein, J., de Cheveigne, S. et al, Astron.Astrophys.79, 256-259 (1979)

3. Tielens, A.G.G.M., Allamandola, L.J., Bregman, J. et al, Astrophys.J. 287, 697-706 (1984)

4. Lacy, J.H., Baas, F., Allamandola, L.J. et al, Astrophys.J. 276, L533-L538 (1984)

5. Léger, A., Astron.Astrophys.123, L271-L275 (1983)

6. Dartois, E., Space Science Reviews, 119, 293 (2005)

7. Gibb, E.L., Whittet, D.C.B., Boogert, A.C.A. and Tielens, A.G.G.M., Astrophys.J. Supp., 151, 35-73 (2004)

8. Dartois, E., Demyk, K., d'Hendecourt, Ehrenfreund, P., Astron.Astrophys., 351, 10661074 (1999)

9. Dartois, E., d'Hendecourt, L., Astron.Astrophys., 365, 144-156 (2001)

10. d'Hendecourt, L., Jourdain de Muizon, M., Astron.Astrophys. 223, L5-L9 (1989)

11. d'Hendecourt, L.B., Allamandola, L.J., Baas, F., Greenberg, J.M., Astron.Astrophys. 152, 130-150 (1985)

12. d'Hendecourt, L.B., Allamandola, L.J., Grim, R.J.A. et al. Astron.Astrophys. 158, 119-134 (1986)

13. De Graauw, T., Whittet, D.C.B., Gerakines ? P.A., et al. Astron.Astrophys., 315, L345-L349 (1996)

14. Hudson, R.L. and Moore, M.H., Astron.Astrophys. 357, 787-792 (2000)

15. Bottinelli, S., Ceccarelli, C., Williams, J.P. and Lefloch, B., Astron.Astrophys. 463, 601-610 (2007)

16. Schutte, W., Khanna, R.K., Astron.Astrophys., 398, 1049-1062 (2003)

17. Raunier, S., Chiavassa, Th., Duvernay, F., et al, Astron.Astrophys. 416, 165-169 (2004)

18. d'Hendecourt, L. and Dartois, E., Ac.Spe. 57, 669 (2001)

19. .Jenniskens, P., Baratta, G.A., Kouchi, A. et al. Astron.Astrophys. 273, 583-600 (1993)

20. Hagen, W., Allamandola, L.J., Greenberg, J.M., Ap.SS, 65, 215 (1979)

21. Hudgins, D.M., Sandford, S.A., Allamandola, L.J. and Tielens, A.G.G.M., Astrophys.J.Supp., 86, 713-870 (1993)

22. Nuevo, M., Auger, G., Blanot, D. and d'Hendecourt, L., OLEB, 38, 37 (2008)

23. Miller, S.L., Science, 117, 528 (1953)

24. Nuevo, M., Bredehöft, J.H., Meierhenrich, et al. Astrobiology, 10, 745-751 (2010)

25. Bernstein, M.P., Sandford, S.A., Allamandola, L.J. et al., Astrophys.J., 454, 327 (1995)

26. De Marcellus, P., PhD. Thesis, Université Paris-Sud, France (2010)

27. Munoz-Caro, G.M., Schutte, W., Astron.Astrophys. 412, 121-132 (2003) 
28. Munoz-Caro, G.M., Meierhenrich, U.J., Schutte, W.A., et al. Nature, 416, 403-407 (2002)

29. Kaiser, R.I., Stockton, A.M., Kim, Y.S. et al., Astrophys.J. 765, 111-118 (2013)

30. Sephton, M.A., Nat.Prod.Rep. 19, 292 (2002)

31. de Marcellus, P., Meinert, C., Nuevo, M. et al. Astrophys.J. 727, L27-L34 (2011)

32. Fukue, T., Tamura, M., Kandori, R. et al, OLEB, 40, 335 (2010)

33. Meierhenrich, U.J. in Aminos Acids and the Asymmetry of Life, Springer (2005)

34. d'Hendecourt, L., Joblin, C., Jones, A., eds., in Solid State Interstellar Matter: the ISO Revolution, EDP Science and Springer Verlag (1999)

35. Pinti, D., L., in Lectures in Astrobiology, Vol 1, Part 1, p.83, Gargaud, M., Barbier, B., Martin, H., Reisse, J. eds., Springer (2006)

36. Commeyras, A., Taillades, J.,Collet, H. et al. OLEB, 34, 35 (2004)

37. de Marcellus, P., Bertrand, M., Nuevo, M., Westall, F., Le Sergeant d'Hendecourt, L. accepted in Astrobiology (2011)

38. Nielsen, P.E., OLEB, 37, 323-328 (2007)

39. Pross, A. in What is Life : when chemistry becomes biology, Oxford University Press, ISBN 978019964101 è (2012)

\section{Acknoweldgements}

.Experiments on ice photochemistry have been performed at IAS and at the UV-CPL line DESIRS on the synchrotron SOLEIL. The authors acknowledge their strong collaboration with Dr. L. Nahon (DESIRS-SOLEIL) and Drs. U. Meierhenrich and C. Meinert for GCxGC-MS analysis of the samples at the LCMBA in Nice. Financial support from the French CNRS program PCMI, the CNES exobiology and the EPOV program are greatly acknowledged. 